\title{
A New Phase for Superfluid Helium-3
}

\section{Confining helium-3 in an aerogel container and subjecting it to a magnetic} field reveals a long-predicted superfluid phase.

\section{By Marric Stephens}

$\square$ uperfluidity is a familiar phenomenon in helium $-4\left({ }^{4} \mathrm{He}\right)$, whose individual atoms are bosons. It can also occur in helium-3 $\left({ }^{3} \mathrm{He}\right)$, when individual (fermionic) atoms pair up to make bosonic complexes called Cooper pairs. Because in ${ }^{3} \mathrm{He}$ these Cooper pairs have nonzero total spin, superfluid ${ }^{3} \mathrm{He}$ has a richer phase space than superfluid ${ }^{4} \mathrm{He}$. Now, Vladimir Dmitriev at the P. L. Kapitza Institute for Physical Problems, Russia, and colleagues have added to that richness, observing the long-predicted, but never-before-seen, superfluid $\beta$ phase of ${ }^{3} \mathrm{He}[1]$.

The main factors that determine the phase of superfluid ${ }^{3} \mathrm{He}$ are its temperature and pressure. But additional factors also play a role. One factor is the presence of a magnetic field, which has previously been shown to split the so-called $A$ phase into two phases that contain Cooper pairs with different spin compositions. Another factor, which also changes the structure of the Cooper pairs, is the symmetry of the geometry in which the superfluid is confined. In a previous study, Dmitriev and

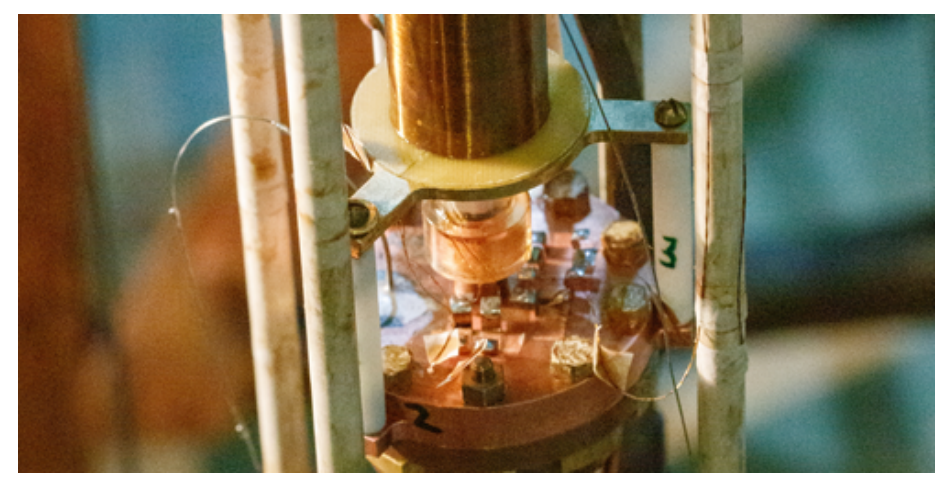

Credit: A. Yudin/P. L. Kapitza Institute for Physical Problems colleagues confined superfluid ${ }^{3} \mathrm{He}$ in an aerogel whose pores were much longer than they were wide. In doing so, they were able to observe the superfluid's so-called polar phase.

The researchers have now studied this anisotropically confined superfluid in a magnetic field. They find that the magnetic field changes the superfluid's phase-transition path. Instead of reaching the polar phase via a single transition as the temperature drops, the superfluid goes through two phase transitions. In between these two transitions it forms a previously unseen $\beta$ phase. The researchers say that their results could provide insight into the behaviors of other Cooper-pair-containing systems, such as unconventional superconductors, quantum gases, and, possibly, neutron stars.

Marric Stephens is a Corresponding Editor for Physics based in Bristol, UK.

\section{REFERENCES}

1. V. V. Dmitriev et al., "Superfluid $\beta$ phase of ${ }^{3} \mathrm{He}$," Phys. Rev. Lett. 127,265301 (2021). 